\title{
Division I Working Group on Future Development of Ground-Based Astrometry
}

\author{
CHAIR: Magda Stavinschi
}

\begin{abstract}
The working group "The Future Development of Ground-Based Astrometry" of the IAU Division 1, founded in 2000, continued its activity for the last triennium. Part of its resultsmeetings or programs - are presented here. The FDGBA web site is http://www.astro.ro/wg
\end{abstract}

\section{Introduction}

As Newsletter No. 1 of the IAU Commission 8 announced, "The post-Hipparcos era has brought an element of uncertainty as to the goals and future programs for all of ground-based astrometry." The WG had as main objectives to identify such programs and make assessments of the whole situation including available instrumentation. As its statutes specifies, it has "to identify scientifically important programs that can be realized using ground-based astrometric or related observations, and to study what kind of modifications, upgrades or additions to the existing instruments should be performed in order to provide useful astronomical information with necessary accuracy, keeping in mind what the future astrometric satellites will contribute."

A final objective that we see for this working group is the identification of programs that could be made on instruments that are either insufficiently used or are working on projects that have no significant value for the present-day astrometry. A major reason for this is that these instruments can be used as they are or with not too expensive modifications to teach students in astronomy how to use telescopes and, at the same time, to contribute to astronomy in a significant way.

The working group has its own web site http://www.astro.ro/wg, updated every time it was necessary.

During its first triennium of activity, FDGBA organized three international meetings (Munich 2001, Bucharest 2002, Sydney 2003).

The IAU Division 1 approved the prolongation of FDGBA activity until the next General Assembly (Prague, 2006) when it will become part of the Commission 8 Astrometry.

\section{Meetings}

FDGBA organized special meetings, offering to the participants a better knowledge of the programs used by the Ground-Based Astrometry today, which could be its contribution in competition with the space missions, and-last but not least - the training of future astrometrists.

In 2004 a special meeting "Astrometry with Small Telescopes" was organized in Bucharest, Rumania, 22-23 October. The main problems discussed during the last meeting were the following:

- Techniques and software on narrow-field CCD astrometry using ground-based telescopes;

- Observations and data processing of radio-stars, asteroids, mutual phenomena of Solar system bodies, KBO's; 
- Theoretical and observational aspects concerning the dynamics of the Solar system;

- Contribution of the ground-based astrometry to the improvement of celestial and local reference systems.

The communications (invited, oral or poster) were presented in the "Proceedings of the Scientific Session ASTROMETRY WITH SMALL TELESCOPES", Bucharest, Rumania, 22-23 October 2004, eds. Magda Stavinschi and Vasile Mioc, as a Supplement of the Rumanian Astronomical Journal, with the financial support of UNESCO-ROSTE.

\section{Training}

The future for astrometric research is very exciting due to the SIM and GAIA astrometric space missions, which will provide 10-20 microarcsecond positions, parallaxes and proper motions for 109 stars. The potential for studies of the structure, kinematics and dynamics of our Galaxy, as well as for the physical nature of stars and the cosmological distance scale, is without equal in the history of astronomy.

Hipparcos and the HST were astrometric successes due only to the dedicated work of specialists in astrometry, who fought to maintain the astrometric characteristics of those satellites and their data pipelines. It is ironic therefore that given this unparalleled treasure trove of data that in two years not one course in astrometry will be taught in the US, leaving all astrometric education to Europe, China and Latin America. Who will ensure the astrometric quality control for the JWT, SIM, GAIA, to say nothing about the current large ground-based facilities, such as the VLT, Gemini, Keck, NOAO, Magellan, the LBT, etc.?

So, we propose a renewal of astrometric education in the universities to prepare qualified scientists for these unique opportunities so that the scientific returns from the investment of several billions of dollars will be maximized. The universities, national and international observatories and agencies should acknowledge their responsibility to hire qualified full time astrometric scientists to supervise existing and planned astronomical facilities so that quality data will be obtained. The funding agencies are providing outstanding facilities. Will the universities, institutes and observatories to assume their responsibilities?

The result of this proposal was the Yale Summer Workshop on Basic Astrometric Methods, July 18-22, 2005, Yale University, New Haven, Connecticut, USA. The main goal was to provide an intensive introduction to the basic methods needed to utilize facilities with advanced astrometric capabilities.

\section{Special programs}

\subsection{WFPDB (K. Tsvetkova and P. Lampens)}

The status of the astrometric wide-field plates in the Wide-Field Plate Database (WFPDB, http://www.skyarchive.org), is published by Tsvetkova, Tsvetkov, Stavinschi, Fresneau and Lampens (2005) in the Proceedings of the International Scientific Session "Astrometry with Small Telescopes", Bucharest, Romania, 22-23 October 2004, eds. Magda Stavinschi and Vasile Mioc, RoAJ Vol. 15, Supplement, 2005.

The WFPDB with its 375 archives, containing more than 2,130,000 plates obtained with professional telescopes of 125 observatories worldwide, is now the basic source of information for the archived observations. Their importance is in connection with analyzing of the long-term variations (sometimes more than a century) of the positions, orbits or dimensions of a lot of celestial objects or their prediscovery history. At present there 
is an on-line access to the descriptive data for 377,687 plates from 92 archives through the WFPDB updated version in the Sofia Sky Archive Data Center (SSADC). Archives of selected digitized plates are under preparation.

It is useless to point out once more the importance of the photographic archives and show the potential of the WFPDB for future astrometric researches. In the WFPDB there are 56 astrographs (telescopes usually dedicated to astrometry) with focal length in the range $0.57-6.90 \mathrm{~m}$, which have produced 65 archives in the period 1891-1995, with 285,658 direct plates and 3,966 objective prism plates. The list of observatories in the WFPDB with telescopes having a scale of about $60 " / \mathrm{mm}$ includes some of the observatories, which have taken part in the Carte du Ciel project: Greenwich, Vatican, Catania, Potsdam, Bordeaux, Toulouse, Uccle and Bouzareah.

The wide-field plate collection of the Royal Observatory of Belgium is a good example of plate archives devoted to astrometry. The last $\mathrm{CdC}$ archive that is prepared for inclusion in the WFPDB is the Uccle archive (600 plates of good quality and starting at epoch 1908).

The conclusions are that the on-line access to the descriptive data for 377,687 plates from 92 archives of the WFPDB updated version in Sofia Sky Archive Data Center (SSADC) offers good opportunities for further astrometric researches-long-term observations for many celestial objects made with suitable telescopes and proposing good quality data. Archives of selected digitized plates being on-line are under preparation. In particular, the CdC plates of good quality still offer great value for any research task aided by the long-term acquisition of astrometric and/or photometric data.

\subsection{Before GAIA (W. Thuillot and M. Stavinschi)}

The aim of this topic was to call for observers and to describe the objectives for a followup program of ground-based astrometric observations to compliment certain observations made by the space project GAIA. This program could, in the long term, relate to all the observational aspects of the GAIA project: photometry, spectroscopy and astrometry. But this paper relates to only the astrometric aspect for solar system objects and intends to call for astrometrists to join a dedicated network to carry out these observations.

On the dedicated web page http://aira.astro.ro/wg/gaia.htm some topics are discussed:

- Context and problems;

- Towards a dedicated network;

- Robotic telescopes;

- Conventional telescopes;

- Constraints;

- Actions to be carried out.

The conclusions are that GAIA will provide many positional measurements of objects of the solar system and numerous new objects will be detected. A follow-up program will be necessary to ensure these objects are not lost and to improve their ephemerides. Organizing a network requires time and different steps have to be done. We call observers interested in this network, and particularly astrometric observers, to join the network now to help follow-up GAIA. Some astrometrists reported their interest to such a kind of program.

\subsection{Others}

In 2003 the international campaign PHEMU 2003 was completed. CCD, photoelectric, and video observations of mutual phenomena of the Galilean satellites were carried out in different observatories of the world. The campaign was coordinated by the IMCCE (Institut de Mécanique Céleste et de Calcul des Éphémerides). In 2003, everything was 
favorable for the observations: high declination of Jupiter and opposition at the best moment. The observations of the mutual phenomena of Jovian satellites are interesting for the dynamical study of the Jovian system but they gave us an experience allowing to improve our observational methods in order to increase the accuracy of the data. A lot or articles were published after.

The main programs done in this period concern the identification and observation of ICRF sources, different objects of the Solar System, or the solar diameter.

Some individual reports could be read on the WG web site http://www.astro.ro/wg.

\section{Acknowledgements}

We are grateful to Professor Jean Kovalevsky for his permanent support.

\section{References}

Stavinschi, M. \& Mioc, V. (eds) 2005, Proceedings of the International Scientific Session "Astrometry with Small Telescopes", held in Bucharest, Romania, 22-23 October 2004, RoAJ, 15, Supplement

Thuillot, W, \& Stavinschi, M., 2005, A Follow-up Program for the Space Astrometry Project GAIA, http://aira.astro.ro/wg/gaia.html

Van Altena, W. F., \& Stavinschi, M., 2005, in "Astrometry in the Age of the Next Generation of Large Telescopes", ASP Conference Series, 2005, P.K. Seidelmann and A.K.B. Monet (eds) 